\title{
Fatores Críticos de Adoção da Tecnologia da Informação (TI) em Saúde e o seu Impacto na Gestão: um estudo exploratório
}

Critical Factors in the Adoption of Information Technology (IT) in Health and its Impact on Management: an exploratory study

\section{Resumo}

progresso em tecnologias da informação em saúde oferece novas oportunidades, bem como novos cenários de aplicação, trazendo benefícios em qualidade dos serviços médicos e redução dos custos de saúde. Porém, a literatura científica relacionada com este tema parece ser ainda muito dispersa e fragmentada, demandando novos estudos. Assim, o objetivo geral deste trabalho é investigar a decisão de adoção de tecnologias informação em saúde, e desenvolver uma lista indicando os fatores críticos que influenciam sua adoção e o impactos na gestão da saúde. Para o desenvolvimento da pesquisa foi adotada a pesquisa Qualitativa através de um estudo de caso em um Organização de Saúde e a Tecnologia analisada foi a implantação do Prontuário Eletrônico. Com relação aos resultados, foram identificados os seguintes fatores críticos de adoção: elaboração de um plano diretor de TI, integração com os sistemas legados, tendências tecnológicas promovendo inovação, envolvimento da alta administração, gestão de projetos com equipes hibridas, plano de capacitação para equipe enfermagem, pressão da concorrência, perfil dos pacientes e a necessidade de novos modelos de negócio. Os principais impactos observados com a adoção dos sistemas foram: melhoria da segurança do paciente, redução do Índices de erro de medicamento, visibilidade das informações.

\section{Abstract}

Progress in health information technologies offers new opportunities as well as new application scenarios, bringing benefits in the quality of medical services and reducing health care costs. However, the scientific literature related to this topic seems to be still very dispersed and fragmented, demanding new studies. Thus, the general objective of this work is to investigate the decision to adopt health information technologies, and to develop a list indicating the critical factors that influence its adoption and the impacts on health management. For the development of the research was adopted the Qualitative research through a case study in a Health Organization and the Technology analyzed was the implementation of Electronic Records. Regarding the results, the following critical adoption factors were identified: elaboration of an IT master plan, integration with legacy systems, technological trends promoting innovation, senior management involvement, project management with hybrid teams, staff training plan nursing, competitive pressure, patient profile and the need for new business models. The main impacts observed with the adoption of the systems were: improvement of patient safety, reduction of drug error indexes, visibility of information.

Keywords: 


\section{INTRODUÇÃO}

De acordo com Leslie e Paradis (2018), o Affordable Care Act (ACA) encorajou, em 2009, o uso da Tecnologia da Informação em Saúde para alcançar melhorias nesta área. O sistema de tecnologia da informação em saúde deve contribuir para a melhoria da qualidade, da eficiência e da eficácia do atendimento em saúde. Assim, como finalidades principais, pode-se afirmar que um sistema de tecnologia da informação deve servir para gerenciar a informação que os profissionais de saúde precisam para desempenhar as atividades com efetividade e eficiência, facilitar a comunicação, integrar a informação e coordenar as ações entre os diversos membros da equipe profissional de atendimento, fornecendo recursos para apoio financeiro e administrativo. Dobrzykowski et al. (2015) mencionam que a coordenação ou a troca de informações no hospital constitui um desafio, pois os processos são intensivos em informações, complexos e exigem várias interações. Neste sentido, a tecnologia da informação pode ter um importante papel como meio de promover melhorias no processo de saúde. Rejeb, et al. (2017) argumentam que muitas organizações de saúde, consideram a aplicação da Tecnologia da Informação como uma chave fundamental para melhorar a gestão de saúde, porém poucos trabalhos analisam os fatores de adoção e o impacto de sistemas de informação em termos econômicos e de qualidade dos cuidados aos pacientes. Elliott (2014) também tem um estudo convergente, mencionando que existem poucas pesquisas que relacionem tecnologia da informação ao custo efetividade em setores individuais hospitalares (Unidade de Terapia Intensiva - UTI - por exemplo), ou procedimentos médicos; além disso a comunidade pesquisadora não tem se preocupado em considerar as características da tecnologia informação em saúde e as relações que vinculam a informação ao seu ambiente para tomada de decisão (SEDIG; NAIMI; HAGGERTY, 2017). Segundo Aceto et al. (2018) o progresso em tecnologias da informação em saúde oferece novas oportunidades, bem como novos cenários de aplicação, trazendo benefícios em qualidade dos serviços médicos e na redução dos custos de saúde. Os autores afirmam que a literatura científica relacionada a este tema parece ser ainda muito dispersa e fragmentada, demandando novos estudos.

Com base nas lacunas discutidas pelos autores citados, elaborou-se a pergunta que norteou o desenvolvimento deste estudo: Como os hospitais estão implantando a Tecnologia da Informação em Saúde? Quais os fatores críticos que influenciam na sua adoção e os resultados obtidos com a tecnologia adotada?

\section{REFERENCIAL TEÓRICO}

Os sistemas de informação na área da saúde podem ser classificados em três tipos de acordo com Hannan, Ball, Edwards (2009):

- O primeiro é composto de sistemas limitados quanto ao objetivo e ao escopo. O mais comum é 
o sistema isolado (stand-alone) direcionado a uma área específica de aplicação.

- O segundo tipo é composto de sistema de informação hospitalar, que, com frequência, consiste de uma rede de comunicação, um componente clínico e um componente administrativo e financeiro.

- O terceiro tipo, sistemas corporativos de informação em saúde, está em expansão nos ambientes de saúde. Tais sistemas capturam e armazenam informações mais completas, provenientes da assistência à saúde continuada realizada por diferentes organizações, usando um modelo integrado de prestação de serviços. Esses sistemas são caracterizados por focar o paciente que está recebendo o cuidado, em diversos setores (por exemplo, ambulatório, unidade de tratamento intensivo, internação de longa permanência), com uma estrutura comum e organizada.

Faber et al. (2017) mencionam que as inovações em TI nas organizações, incluindo hospitais, constituem um processo de decisão que envolve duas partes. Em primeiro lugar, uma decisão de adoção formal para comprar, adotar ou adquirir uma inovação e disponibilizá-la à organização. Seguido por decisões de usuários locais (por exemplo, profissionais da área médica) sobre se realmente usarão a inovação e como. Este tipo de tecnologia traz muitos desafios e, portanto, compreender a sua adoção torna-se um fator importante para alavancar o potencial de resultados na gestão da saúde. Um meio de compreender a adoção de novas tecnologias e os respectivos fatores críticos pode ser por meio do framework (Technology-Organization-Environmental) o qual será discutido no tópico 2.1.

\subsection{Framework Technology-Organization-Environmental (TOE)}

Tornatzky e Fleischer (1990) propuseram um framework denominado TOE, o qual é visto por grande parte dos estudos de adoção de inovação tecnológica como a estrutura mais adequada para compreender a adoção da tecnologia da informação em um contexto organizacional (THOMAS et al., 2008; CHANG et al., 2006, OLIVEIRA et al. 2011). O framework TOE explica que três diferentes contextos influenciam nas decisões de adoção de novas tecnologias, sendo eles: o contexto tecnológico, o contexto organizacional e o contexto ambiental, conforme apresentado na Figura 1. O framework TOE como originalmente apresentado, e mais tarde adaptado em estudos de adoção de TI, fornece um quadro analítico útil que pode ser usado para estudar a adoção e assimilação de diferentes tipos de inovação de TI.

Os fatores tecnológicos descrevem as características do ambiente tecnológico, que podem influenciar a difusão dos sistemas na organização. Estas características incluem diversas dimensões da tecnologia, desde a vantagem relativa à qualidade da informação do sistema, que aumentam a difusão da tecnologia da informação na organização. Os fatores organizacionais referem-se ao escopo, recursos e tamanho da organização e a dimensão ambiente refere-se ao contexto em que a empresa 
desenvolve a sua indústria, concorrentes e relações com o governo (TORNATZKY; FLEISCHER ,1990).

Figura 1 - A estrutura TOE

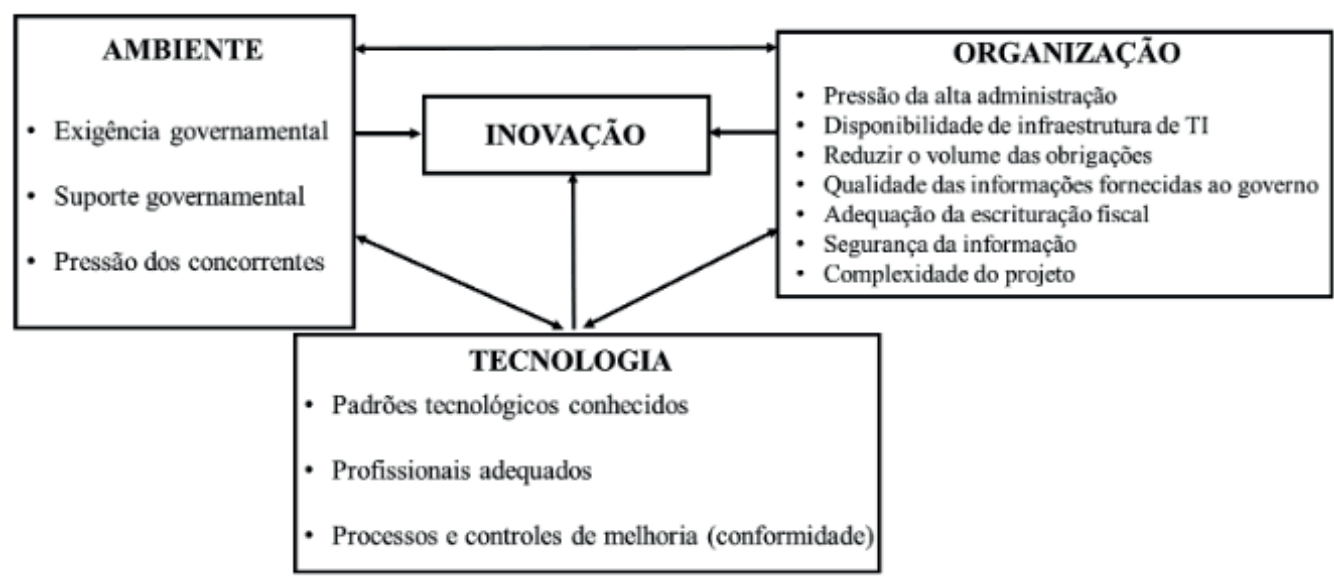

Fonte: Adaptado de Baker, 2011.

De acordo com Handayani et al. (2017) a gestão hospitalar e os desenvolvedores de TI devem ter mais compreensão sobre os fatores não-tecnológicos para melhor planejar a sua implementação. $\mathrm{O}$ envolvimento da gestão é fundamental para assegurar que sua implementação tenha impacto e proporcione benefícios para os usuários, bem como para os hospitais.

Alkraij et al. (2016) utlizaram o framework TOE e efetuaram uma pesquisa em organizações de saúde identificando dezessete fatores relacionados com a adoção de tecnologia da informação em saúde e com os dados de saúde disponíveis nessas instituições. Os resultados demonstraram uma lista de fatores-chave de diferentes aspectos que afetam a decisão de adoção de padrões de dados de saúde nas organizações. Os fatores tecnológicos são: complexidade e compatibilidade dos padrões de dados de saúde, infraestrutura de TI, incertezas em relação aos custos, integração de sistemas e aprimoramento do uso de sistemas avançados. Os principais fatores organizacionais são: a falta de políticas e procedimentos adequados e plano de gestão da informação, resistência à mudança, e à análise de dados. Os principais fatores ambientais são: a falta de um órgão regulador nacional e um plano de troca de dados, falta de um sistema nacional de saúde e a carência de profissionais.

Angst e Agarwal (2009) mencionam que no contexto emergente de digitalização de saúde, registros eletrônicos constituem um avanço tecnológico significativo da forma que a informação médica é armazenada, comunicada e processada pelas várias partes envolvidas na entrega dos cuidados de saúde e esta característica gera desafios relacionados com a sua adoção por parte das organizações de saúde, sendo, portanto, muito pertinente aplicações de frameworks como o TOE para uma melhor compreensão da adoção de novas tecnologias na área da saúde. 


\subsection{Impactos da adoção de Tl em Saúde}

Lilford et al. (2014) mencionam que a partir da avaliação tecnológica é possível fazer uma comparação entre as tecnologias de informação em saúde hospitalar e de que maneira o uso dessas tecnologias traz benefícios para o setor hospitalar, permitindo avaliar a implementação, a adoção, e a eficácia dos sistemas de publicação eletrônica à medida em que são introduzidos em hospitais. A busca por maior eficiência na gestão de saúde levou a inúmeras propostas para aumentar a adoção de TI em saúde. Dois estudos realizados, um pelo RAND Corporation e outro pelo Centro de Informação Technology Leadership (CITL), estimaram que cerca de US\$ 80 bilhões em economia anual líquida é potencialmente atribuída a essa tecnologia (ORZAG, 2008). Freedman et al. (2018) discutiram em seu estudo os impactos relacionados à adoção de tecnologia da informação em saúde nos registros médicos eletrônicos (EMR's). A pesquisa teve foco nos indicadores de segurança do paciente. Os autores mencionaram evidências que os $E M R$ 's reduzem a probabilidade de eventos adversos relacionados com a segurança do paciente, particularmente para pacientes menos complexos.

Wang et.al (2018) mencionam que alguns pesquisadores sugerem um desempenho financeiro positivo, com base em estimativas de savings obtidos com a adoção de tecnologias como os $E M R$ 's.

Segundo Sedig, Naimi, Haggerty (2017), nos últimos anos, a adoção da tecnologia de informações em saúde (HIT-health information technology) teve um crescimento considerável, contribuindo para a comunicação médico-paciente; bases tecnológicas para o aprendizado e melhoria institucional.

\section{PROCEDIMENTOS METODOLÓGICOS}

A abordagem metodológica adotada para esta pesquisa é a qualitativa, operacionalizada por meio de um estudo de caso. De acordo com Voss et al. (2002) e Yin (2005) esta é uma escolha adequada quando o objetivo é compreender um fenômeno que ocorre dentro de um determinado contexto e o pesquisador não possui nenhuma influência sobre o meio, além de procurar responder a questões do tipo "Por que" e "Como". Esta pesquisa está de acordo com estas premissas, uma vez que o objetivo é investigar como organizações de saúde estão implantando as tecnologias de informação em saúde.

\subsection{Objeto de Estudo}

O objetivo geral deste trabalho é o de investigar a decisão de adoção de tecnologias da informação em saúde, e desenvolver uma lista que indique os fatores críticos que influenciam na sua adoção e os impactos na gestão da saúde. Para o desenvolvimento da pesquisa 
adotou-se uma abordagem qualitativa por meio do método de estudo de caso em uma instituição de saúde.

\subsection{Procedimentos de Coleta de Dados}

Trata-se de um polo de saúde com mais de 1.000 leitos, 7.000 colaboradores e 3.000 médicos; a instituição atende mais de 1,8 milhão de pessoas por ano, sendo referência no atendimento médico-hospitalar em diversas especialidades. Como instituição sem fins lucrativos, a receita gerada pelo atendimento a pacientes particulares e de convênios, somada às doações feitas pela sociedade, são integralmente investidas no aprimoramento da qualidade assistencial.

\subsection{1 Área TI e Ferramentas Implantadas}

A TI responde diretamente ao CEO do hospital e conta com 103 colaboradores: 52 pessoas fazem parte da área de sistemas, que está dividido em três gerências:

- Assistencial: que cuida de sistemas voltados ao atendimento ao paciente (incluindo do prontuário eletrônico);

- Back office: sistemas voltados para as áreas administrativas como compras, financeira, RH, dispensação, farmácia, estoques;

- Sistemas de SADT (Sistemas de Apoio para Diagnósticos e Terapia): com os sistemas de imagens, sistemas para hemodiálise, sistemas para os laboratórios, para sepsia etc.

E o restante da equipe faz parte da infraestrutura, tendo duas supervisões, uma cuidando do service desk, que ainda é interno dentro da organização estudada, tendo 31 pessoas, e o restante dentro da área de infraestrutura cuidando de redes, servidores, banco, serviços InCloud, e segurança.

A implantação do Prontuário Eletrônico foi uma das tecnologias da informação em saúde que os gestores da organização pesquisada mencionaram como destaque.

A coleta de dados foi obtida por intermédio de entrevistas em profundidade com os responsáveis pela implementação do Prontuário Eletrônico. A forma de condução das entrevistas foi semiestruturada para permitir que os entrevistados pudessem ter liberdade de apresentar os seus pontos de vista e as experiências de sua organização com o tema adoção de tecnologias de informação em saúde. As entrevistas foram gravadas e transcritas. Relatórios contendo os principais pontos das entrevistas foram gerados e enviados para os entrevistados para que pudessem fazer observações adicionais.

\subsection{Procedimentos de Análise de Dados}

Com base nas entrevistas conduzidas com profissionais, relatórios e observações foram levanta- 
dos os fatores críticos de implantação do Prontuário Eletrônico a partir do framework TOE e o respectivo impacto na gestão da saúde conforme mencionado no objetivo geral do estudo.

\section{RESULTADOS}

\subsection{Tecnologia}

A CEO do hospital destacou a importância de ter um plano diretor de TI que determine as linhas de atuação, os tipos de ferramentas tecnológicas, um escopo básico ou mínimo para as questões de processos de segurança, que são fatores importantes para a adoção de novas tecnologias.

Para que a implantação do prontuário eletrônico do paciente fosse realizada, de maneira a atingir as expectativas, houve a necessidade de avaliar a compatibilidade e executar a integração com as ferramentas já existentes no hospital. Um exemplo dado foi com relação à prescrição eletrônica, que demandava uma solicitação de farmácia para dispensação de um produto ou cliente; nesse caso foi necessário integrar e compatibilizar com outras ferramentas disponíveis no hospital para permitir que o médico realizasse a prescrição no prontuário eletrônico, pois a partir disso era gerado uma movimentação de estoque na unidade que posteriormente era adicionado a um consumo, a um Demonstrativo de Resultado (DRE), e a um processo contábil de gestão financeira.

Além do exemplo mencionado, também foi necessário avaliar a compatibilidade e a integração das tecnologias existentes para ferramentas de controle de acesso (médicos cadastrados nos sistemas com acesso ao processo de entrada física dentro hospital) e ferramentas de laboratório, de forma que os resultados ou pedidos de laboratório fossem apresentados aos médicos durante o cuidado do paciente.

Para que esse modelo de integração fosse realizado dentro das melhores práticas foi adotado uma ferramenta de barramento tornando a tecnologia capaz de integrar os sistemas do hospital.

Com relação aos desafios enfrentados para a adoção da tecnologia, o destaque se dá no quesito de orçamento e não na infraestrutura tecnológica, pois tomada a decisão da implantação do prontuário eletrônico foi necessário adquirir uma ampla quantidade de equipamentos; houve então um processo de revisão da infraestrutura (servidores, redes, desktops).

\subsection{Organização}

A motivação organizacional para a adoção da ferramenta se deu por fatores como:

a) aumento de receita e redução de custos por ser um processo otimizador;

b) eliminação de riscos de desenvolvimento interno; 


\section{SI}

c) preocupação da organização com relação à segurança assistencial do paciente;

d) satisfação do cliente (paciente e médico).

Os principais custos associados à adoção da ferramenta foram relacionados ao pagamento de licenças, infraestrutura, processos de infraestrutura de reforma de ambientes. Para comportar o número de equipamentos necessários (espaço para comportar o sistema de prontuário eletrônico que está associado a um processo de ciclo fechado do medicamento, onde a enfermeira transporta um carrinho de medicação em cada leito e realiza o processo de leitura da pulseira do paciente e do medicamento, de forma que todo o trajeto seja rastreado) foi necessário adquirir e locar leitoras de código de barras, espaços físicos para os carrinhos que comportam os notebooks para que pudessem ser estacionados e ter as suas baterias carregadas, mão de obra específica de TI com expertise no processo de adoção, processos de gestão de mudanças e treinamento.

Com relação ao treinamento, participaram deste processo 9.000 pessoas entre colaboradores (7.500) e médicos (corpo clínico). Como meio de evitar conflitos frente a nova tecnologia foram realizadas as seguintes atividades entre os usuários:

a) envolver os profissionais que utilizariam o sistema na sua escolha (criando compromisso, comprometimento, escolha realizada em conjunto);

b) levantar quais eram as necessidades das áreas, verificando se o profissional responsável por implantar as mudanças conhecia a área e seus processos;

c) implantar a metodologia de multiplicadores, onde colaboradores foram treinados e estes treinaram outros, e assim por diante, facilitando o processo de mudança.

No caso da equipe de TI, que trabalhou na adoção do processo em si, a alternativa usada foi: ao invés de criar duas equipes (uma de projeto e uma equipe para sustentar os sistemas que existiam), foram criadas equipes híbridas; profissionais da própria empresa e que davam sustentação aos sistemas antigos participaram do projeto, e, o inverso também ocorreu, os novos funcionários contratados efetivamente para o projeto participaram da sustentação da empresa, fazendo com que o projeto fosse de todos e não um projeto específico de um grupo da área de TI.

A falta de políticas ou procedimentos adequados não impediram a implantação da ferramenta, porém dificultaram, pois, a falta destes acarretou em maiores gastos de tempo e maior dificuldade no processo de implantação.

\subsection{Ambiente}

Existe uma pressão do ambiente externo, portanto o hospital procura analisar seus concorrentes e com isso, o investimento em tecnologias é uma consequência de forma que a organização possuindo o prontuário eletrônico com selo de qualidade possa se equiparar aos melhores hospitais do Brasil e 
do exterior. Outros fatores externos destacados para a adoção de novas tecnologias foram: a necessidade de novos modelos de negócio, que possam olhar melhor para a saúde do paciente; a tecnologia permite uma melhor coleta de informações para predições de problemas de saúde; e a possibilidade de trabalhar juntamente com a operadora de saúde para fazer um novo modelo onde hospital, operadora e paciente se beneficiam de forma mútua.

De acordo com a entrevistada, não existem questões relacionadas à legislação, porém observa-se a falta de profissionais habilitados para operar o sistema, ou seja, observa-se problemas de formação acadêmica tornando um inibidor da adoção de novas tecnologias voltadas para a saúde, pois as escolas médicas e de enfermagem não formam o aluno para lidar com as novas tecnologias de informação.

\subsection{Impactos com a Implantação do Prontuário Eletrônico}

A organização destacou que uma atuação de forma consistente sobre estes fatores críticos de implantação pode trazer impactos relevantes tanto para a organização como para o paciente. Os impactos levantados e evidenciados nas entrevistas e relatórios estão mencionados a seguir:

- Melhoria da segurança do paciente;

- Índices de erro de medicamento tiveram uma baixa significativa;

- Número de devoluções diminuiu porque quando a prescrição é feita pelo médico há uma otimização do processo de dispensação dos produtos;

- Visibilidade de informações; a própria simultaneidade da visualização de um prontuário pela equipe multidisciplinar;

- A qualidade da documentação do Prontuário Eletrônico no sentido de amplitude e direcionamento em cima das informações que são requeridas;

- Criação de uma base para dados epidemiológicos.

Além da possibilidade da implantação de ferramentas de apoio a decisão auxiliando o médico, ferramentas de apoio em relações a doses limites, em relação a alergias e interações medicamentosas.

\section{DISCUSSÕES}

A aplicação do modelo teórico TOE conforme discutido por Baker (2011) e Alkraiji (2016) apresenta-se como uma abordagem que auxilia no entendimento das tecnologias implantadas, sendo útil para examinar os fatores que afetam a assimilação da TI. Combinar os fatores críticos de adoção e os impactos obtidos da tecnologia implantada, permite um direcionamento para obtenção de melhores resultado em investimentos em Tecnologia da Informação em saúde. Alinhado com os autores Angst e Agarwal (2009), o prontuário médico constituiu um avanço tecnológico significativo na 
forma que a informação médica é armazenada, comunicada e processada.

Conforme mencionado, a implantação do prontuário eletrônico representou um desafio, pois várias mudanças foram necessárias. O estudo de caso indicou os fatores críticos de adoção do prontuário eletrônico (sumarizados na Tabela 1) e os impactos observados com a implantação deste sistema, como melhoria da segurança do paciente, redução do índice de erro na medicação, possiblidade de implantação de ferramentas de apoio a decisão, dentre outros impactos mencionados.

Tabela 1 - Fatores críticos de adoção de TI

\begin{tabular}{ll}
\hline & Fatores críticos de adoção \\
\hline Tecnologia & - Elaboração do Plano Diretor de TI \\
& - Integração com os sistemas legados \\
& - Tendências Tecnológicas \\
\hline Organização & - Envolvimento da alta administração \\
& - Gestão de Projetos com equipes híbridas \\
& - Plano de capacitação para equipe de \\
& enfermagem e de médicos \\
& - Treinamento diferenciado para minimizar \\
& as resistências dos usuários \\
& \\
\hline Ambiente & - Pressão da concorrência \\
& - Perfil dos pacientes \\
& - Necessidade de novos modelos de negócio \\
\hline
\end{tabular}

Fonte: Elaboração dos autores.

Este estudo forneceu várias tendências e insights práticos sobre a adoção de uma Tecnologia da Informação em hospitais que pode servir de base, fornecendo orientações práticas e estratégias, para tomadores de decisão.

\section{CONCLUSÕES}

O cuidado com a saúde representa um importante desafio social e econômico que cada país enfrenta. $\mathrm{O}$ aumento do custo da assistência médica, envelhecimento e o constante crescimento populacional influenciam as demandas de saúde e ditam a necessidade de novas e mais avançadas soluções científicas. Este cenário demanda a implantação de novas tecnologias em saúde, que contribuam com a segurança do paciente, auxiliem o processo de tomada de decisão ao médico, e que suportem o processo de gestão da saúde. O framework TOE fornece um quadro analítico útil que pode ser usado para estudar a adoção e assimilação de diferentes tipos de inovação de TI. Compreender os 
fatores que têm efeitos significativos sobre o sucesso da adoção de novas tecnologias contribui do ponto de vista prático, suportando o processo decisório dos gestores, bem como para a formulação de políticas para implantar soluções eficazes para médicos e pacientes. Este estudo consiste em uma pesquisa exploratória demandando pesquisas futuras, como efetuar estudos de caso em outros hospitais, estudos longitudinais sobre a adoção de outras ferramentas de Tecnologia da Informação em Saúde e os seus respectivos impactos.

\section{Referências}

ACETO, G.; PERSICO, V.; PESCAPÉ, A. The role of Information and Communication Technologies in healthcare: taxonomies, perspectives, and challenges. Journal of Network and Computer Applications, v. 107, 2018.

ALKRAIJI, A. I.; JACKSON, T.; MURRAY, I. Factors impacting the adoption decision of health data standards in tertiary healthcare organisations in Saudi Arabia. Journal of Enterprise Information Management, v. 29, n. 5, p. 650-676, 2016.

ANGST, C. M.; AGARWAL, R. Adoption of electronic health records in the presence of privacy concerns: The elaboration likelihood model and individual persuasion. MIS quarterly, v. 33, n. 2, p. 339-370, 2009.

BAKER, J. The Technology-Organization-Environment Framework. Information Systems Theory, p. 231-245, 2011.

CHANG, I., HWANG, H., YEN, D. AND LIAN, J. “Critical factors for adopting PACS in Taiwan: views of radiology department directors”. Decision Support Systems, v. 42, n. 2, p. 1042-105, .2006 .

DOBRZYKOWSKI, D. D. Understanding information exchange in healthcare operations: Evidence from hospitals and patients. Journal of Operations Management, v.36, p. 201-214, 2015.

ELLIOTT R .L. A; PUTMAN, K. D; FRANKLIN, M. Cost Effectiveness of a Pharmacist-Led Information Technology Intervention for Reducing Rates of Clinically Important Errors in Medicines Management in General Practices. Pharmaco Economics, v. 32, n. 6, p. 573-590, 2014. 
FABER, S.; VAN GEENHUIZEN, M.; DE REUVER, M. Health adoption factors in medical hospitals: A focus on the Netherlands. International Journal of Medical Informatics, v. 100, p. 77-89, 2017.

FREEDMAN, S.; LIN, H.; PRINCE, J. Information technology and patient health: Analyzing outcomes, populations, and mechanisms. American Journal of Health Economics, v. 4, n. 1, p. 51-79, 2018.

HANNAH K. J.; BALL, J. M.; EDWARDS, M. J. A. Introdução à informática em enfermagem. 3a ed. Porto Alegre: Artmed; 2009.

HANDAYANI, P. W. et al. Acceptance model of a hospital information system. International Journal of Medical Informatics, v. 99, p. 11-28, 2017.

LILFORD, et al. Protocol for evaluation of the cost-effectiveness of ePrescribing systems and candidate prototype for other related health information Technologies. BMC Health Services Research ,2014.

ORSZAG, P. R. Evidence on the costs and benefits of health information technology. In: TESTIMONY BEFORE CONGRESS. 2008. Disponível em: https://www.cbo.gov/sites/default/files/ 110th-congress-2007-2008/reports/05-20-healthit.pdf. Acesso em 08 mai. 2018.

OLIVEIRA, T.; MARTINS, M. F. Literature review of information technology adoption models at firm level. The Electronic Journal Information Systems Evaluation, v. 14, n. 1, p. 110-121, 2011.

REJEB, O., PILET, C.; HAMANA, S. X.; DURAND, T. A. Performance and cost evaluation of health information systems using micro-costing and discrete-event simulation. Health Care Management Science, p. 1-20, 2017.

SEDIG, K.; NAIMI, A.; HAGGERTY, N. Aligning Information Technologies With Evidence-Based Health-Care Activities: A Design And Evaluation Framework. Humam Technology, v. 13, n.2, p. 180-215, 2017.

THOMAS, J.; PROBETS, S.; DAWSON, R.; KING, T. A step towards the adoption of standards 
within the UK Ministry of Defence. Journal of IT Standards \& Standardization Research, v. 6, n. 1, p. 55-69, 2008.

VOSS, C.; TSIKRIKTSIS, N.; FROHLICH, M. Case research in operations management. International Journal of Operations \& Production Management, v. 22, n. 2, p. 195-219, 2002.

WANG, T.; WANG, Y.; MCLEOD, A. Do health information technology investments impact hospital financial performance and productivity? International Journal of Accounting Information Systems, v. 28, p. 1-13, 2018.

YIN, R. K., Ed. Estudo de Caso: Planejamento e Métodos, 3a ed. 2005. 